\title{
Análisis de riesgo en Puente peatonal tipo armadura utilizando sensores
} Risk analysis in truss-type pedestrian bridge using sensors

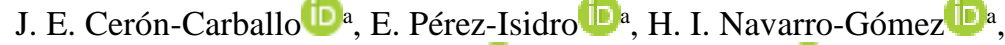 \\ C. Rodríguez-Álvarez ${ }^{\mathrm{DD}}$, G. Ramos-Torres $\mathrm{iD}$ b \\ a Área Académica de Ingenieria y Arquitectura, Universidad Autónoma del Estado de Hidalgo, 42184, Mineral de la Reforma, Hidalgo, México. \\ ${ }^{b}$ Facultad de Ingenieria Civil, Universidad del Estado de Sonora, 83000, Hermosillo, Sonora, México
}

\section{Resumen}

En este trabajo se presenta el análisis del riesgo de la estructura de un puente peatonal ubicado en la colonia Matilde Municipio de Pachuca de Soto, Estado de Hidalgo, México, fabricado en dos etapas constructivas, la primera formada por columnas y trabes de concreto armado y la segunda formada por una estructura metálica, ambas estructuras son sometidas a fuerzas permanentes, variables y accidentales, las cuales producen que la estructura propia del puente interactúe en los nodos de unión de las dos etapas y muestre desplazamientos tridireccionales, de tal forma, que en este documento se determina la magnitud de las aceleraciones de los nodos que interactúan entre las dos estructuras mostrando valores que indican reacciones cíclicas, por consiguiente, para determinar el riesgo se identifican las condiciones de movimiento del puente mediante la utilización de sensores que detectan las aceleraciones, encontrando distorsiones cuyos resultados descartan la intervención de rehabilitación general o local.

Palabras Clave: Análisis estructural, Riesgo Estructural, Puente Peatonal, Distorsión, Acelerómetro, Desplazamiento, Dinámica Estructural.

\begin{abstract}
This paper presents the risk analysis of the structure of a pedestrian bridge located in the Matilde neighborhood, Municipality of Pachuca de Soto, State of Hidalgo, country of Mexico, manufactured in two construction stages, the first one consisting of columns and concrete beams. reinforced and the second formed by a metal structure, both structures are subjected to permanent, variable and accidental forces, which cause the structure of the bridge itself to interact at the junction nodes of the two stages and show tridirectional displacements, in such a way, This document determines the magnitude of the accelerations of the nodes that interact between the two structures, showing values that indicate cyclical reactions, therefore, to determine the risk, the conditions of movement of the bridge are identified through the use of sensors that detect the accelerations, finding distortions whose results rule out the general intervention or local.
\end{abstract}

Keywords: Structural Analysis, Structural Risk, Pedestrian Bridge, Distortion, Accelerometer, Displacement, Structural Dynamics.

\section{Introducción}

Un puente peatonal se construye principalmente para que los transeúntes puedan cruzar sin peligro una carretera vehicular de alta velocidad, en este componente urbano las personas suben por medio de escaleras dispuestas en los extremos, y cruzan la carretera caminando por la pasarela del puente, la cual está construida con una losa de concreto armado y las escaleras son construidas por escalones de concreto apoyados en dos alfardas de acero estructural, por otro lado, en los laterales de la pasarela se coloca un barandal de perfiles estructurales ligeros, que funcionan como medio de protección durante el caminar (Capra, 2005) (Zheng, 2018).

La estructura de un puente peatonal es construida con tres columnas de concreto armado (una central y dos en los extremos), ellas sirven de apoyo para colocar las trabes, formando dos marcos, por consiguiente; las columnas, las

\footnotetext{
*Autor para la correspondencia: jesus_ceronc@uaeh.edu.mx

Correo electrónico: jesus_ceronc@uaeh.edu.mx (Jesús Emmanuel Cerón-Carballo), eber_perez@uaeh.edu.mx (Eber Pérez-Isidro), humberto_navarro@uaeh.edu.mx (Humberto Iván Navarro-Gómez), profe_7479@uaeh.edu.mx (Cutberto Rodríguez-Álvarez), Gilberto.ramos@unison.mx (Gilberto Ramos-Torres) 
escaleras, las trabes y la pasarela forman el procedimiento de fabricación en la primera etapa constructiva (Li, 2014).

Las uniones entre la pasarela y las columnas forman tres nodos importantes, en donde se desarrolla la continuidad estructural, en estos nodos se presentan reacciones con tal magnitud como se requiera por las diversas cargas, esta reacción se le nombrará respuesta dinámica con restricciones [RDR], por otro lado, los claros de la pasarela lo forman vigas simplemente apoyadas, en cuyo diagrama de cortantes presenta las reacciones más desfavorables en los extremos del claro, donde se presenta la RDR y cuyo diagrama de momentos presenta sus máximos valores en el centro de los claros, por tanto, en estos dos puntos los llamaremos puntos de respuesta dinámica con libertad de movimiento [RDL] (Salenko, 2021).

La respuesta dinámica se origina cuando los materiales de cada estructura del puente, presenta reacciones tridireccionales en tiempo real correspondientes a los efectos de las cargas impuestas al puente, por consiguiente, la RDR tendrán restricción de movimiento en la dirección donde existe apoyo y los grados de libertad disminuirán, en caso contrario se presentará libertad de movimiento RDL (SETRA, 2006).

La segunda etapa constructiva se integra al momento de fabricar una estructura espacial formada por perfiles estructurales ligeros unidos entre sí con cordones de soldadura (Figura 1). Los nodos de esta estructura realizan funciones específicas por que las reacciones dinámicas internas son de carácter unidireccional, por consiguiente, la continuidad estructural es de forma axial, cuyos efectos son reacciones de tensión y de compresión. Esta etapa se convierte en una carga permanente y las fuerzas dinámicas que afectan a esta estructura crean RDR en los puntos de unión (Alhassan, 2020).

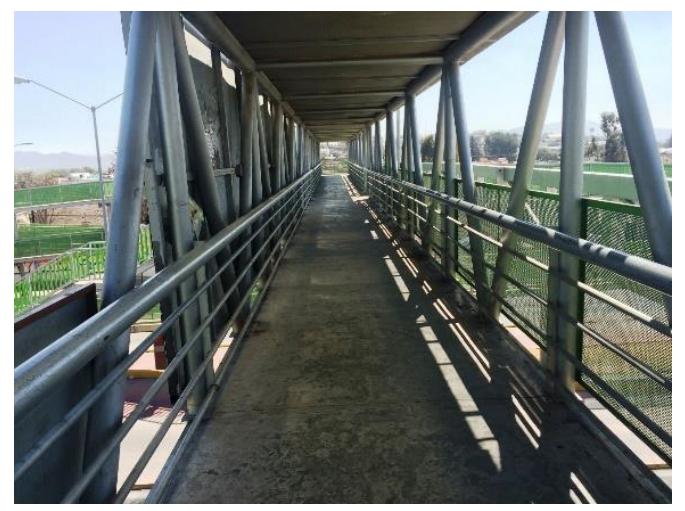

Figura 1: Imagen de la pasarela del puente peatonal. (Fuente: Documental propio tomado en campo)

Las cargas sometidas al puente son de tres índoles, la primera son cargas del peso propio de los materiales de la estructura, y las cargas de transito de los peatones denominadas cargas vivas, ambas dentro de la clasificación de cargas permanentes. La segunda son las cargas variables provocadas por las corrientes de viento y lluvia o su combinación y las terceras son las cargas accidentales que provienen del impacto o de las fuerzas de interacción del suelo-estructura (Budipriyanto, 2015).

\subsection{Análisis de riesgo}

El riesgo estructural es sinónimo de peligro y antónimo de seguridad, los síntomas más comunes es la inestabilidad estructural, este efecto comienza con la perdida de rigidez y se incrementa con las cargas cíclicas, en el análisis estructural del puente se define la vulnerabilidad estructural, como un factor de riesgo controlado (Shi, 2018), es decir, se calcula el valor esperado del daño como una magnitud del movimiento que puede producir la intensidad del fenómeno amenazante, una vez conocido este valor cualquier incremento representa un daño y un incremento del riesgo de perder la estabilidad, cuando la magnitud del riesgo es mayor al reglamentario, es necesario la intervención en la estructura del puente a fin de restablecerla y decrementar las distorsiones (Ali, 2020) (Feldmann, 2010).

\subsection{Acelerómetro}

El acelerómetro es un instrumento que ayuda a determinar la cantidad de movimiento en un nodo, esto permite determinar la magnitud de los desplazamientos en los puntos donde se denoto la RDR y la RDL, este instrumento portátil capta la información en tiempo real (Gheitasi, 2016), la almacena o la puede enviar a un ordenador.

La función del instrumento es calibrado comparando la información recepcionada con la información regulada en laboratorio, controlando métricamente los desplazamientos, para la presente investigación se realiza la calibración de cinco instrumentos, estos tienen una precisión reglamentada (Alhassan, 2020), cada instrumento será denotado con la letra "S". y se distinguirá uno de otro considerando su número de serie (Ali, 2020) (Banerjee, 2020).

\subsection{Normatividad}

Las normas aplicables al estudio y análisis de puentes peatonales son aquellas que regulan las cargas estáticas y las cargas dinámicas, sus efectos y la estabilidad estructural de sus componentes, en este estudio se analizan las cargas dinámicas para establecer criterios de comparación en base a las magnitudes establecidas en los códigos europeos para el estudio de la vibración de estructuras de acero inducida por el hombre HIVOSS, y el codigo de Francia SETRA, asi como las especificaciones American Institute of Steel Construction AISC (Banerjee, 2020) (Gheitasi, 2016) (Feldmann, 2010).

\section{Materiales y Métodos}

La magnitud de la RDR y la RDL varia, denotando movimiento en los puntos con efectos más desfavorables denotados como se muestra en la Figura 2, por la misma dinámica de las cargas impuestas a la estructura del puente, estas cargas cambian de magnitud por su origen propio de ser 
dinámicas, por lo tanto, la magnitud de la RDR y RDL deberán detectarse como un cambio de posición.

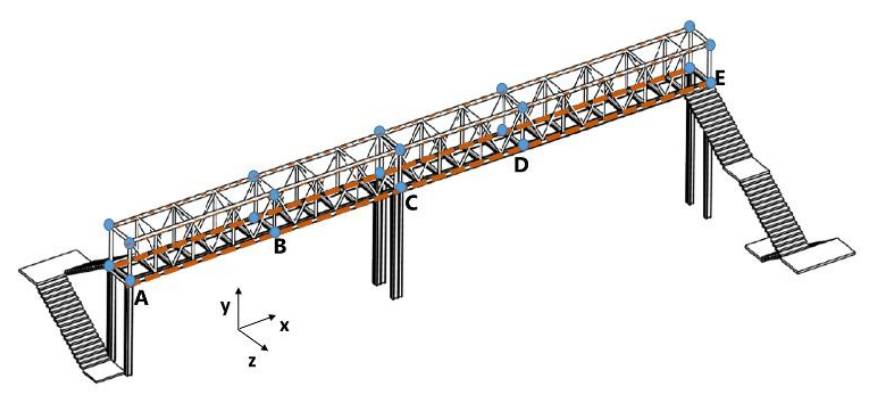

Figura 2: Representación gráfica del puente peatonal. (Fuente: Elaboración propia)

La masa de la estructura del puente se ubica en la posición inicial $\left[\mathrm{x}_{0}, \mathrm{y}_{0}, \mathrm{z}_{0}\right]$ al someterse a una carga cambia a la posición $\left[\mathrm{x}_{1}, \mathrm{y}_{1}, \mathrm{z}_{1}\right]$, y como la carga es dinámica, por consecuencia, tendremos cambios de posición dinámicos, por tanto, los denotaremos como cambios de posición en el dominio temporal $\left[\mathrm{x}_{\mathrm{t}}, \mathrm{y}_{\mathrm{t}}, \mathrm{z}_{\mathrm{t}}\right]$, el desplazamiento es la diferencia de posición " $\mathrm{u}_{(\mathrm{x}, \mathrm{y}, \mathrm{z})}$ " $\left(\left[\mathrm{x}_{1}-\mathrm{x}_{0}\right],\left[\mathrm{y}_{1}-\mathrm{y}_{0}\right],\left[\mathrm{z}_{1}-\mathrm{z}_{0}\right]\right)$ (Alhassan, 2020).

\subsection{Método de Instrumentación}

Se identifican los puntos de la estructura donde se colocarán los sensores, siendo estos los más desfavorables, donde se presenta la RDR, son los puntos de control denotados con las letras A, C y E, la RDL, son los puntos de control denotados con la letra B y D, la orientación de los sensores (SETRA, 2006), se referencia de acuerdo a los ejes cartesianos indicados en la Figura 2.

\subsubsection{Sensores}

Los sensores utilizados son acelerómetros que guardan la información medida en una memoria interna (Budipriyanto, 2015), se colocan en la posición de los puntos de control, este instrumento toma datos y los enlista en una hoja de cálculo con formato "CSV", estos datos son la magnitud de la aceleración y el instante donde fue captada, dichos instrumentos cuentan internamente con un giroscopio y un reloj, digitalmente relaciona tiempo-aceleración, su resolución y rangos para el control son mostrados en la Tabla 1.

Tabla 1: Sensores utilizados en la medición de la aceleración (Fuente: Instrumental propio adquirido)

\begin{tabular}{c|clll} 
SENSOR & CONTROL & MODELO & RANGO & RESOLUCIÓN \\
\hline S-1 & A & Ism6ds3 & 78.4532 & 0.0095768070 \\
S-2 & B & MTK & 13.0000 & 0.0039062500 \\
S-3 & C & LSM6DS3 & 78.4532 & 0.0023956299 \\
S-4 & D & K2HH & 39.2266 & 0.0011971008 \\
S-5 & E & LSM6DS3 & 78.4532 & 0.0005950928
\end{tabular}

\subsection{Método de control de información.}

Los instrumentos son colocados en el centro de la pasarela donde se indican los puntos de control mostrados en la Figura 2 , se realizó una programación para colocar los sensores y activarlos para que inicien a captar información de acuerdo a las magnitudes de las cargas actuantes, esta programación es definida considerando la estadística de las cargas actuantes, debido a las magnitudes más representativas y a las combinaciones de carga (Banerjee, 2020).

\subsection{Estadística de cargas actuantes}

Las fuerzas que provocan las cargas desarrollan desplazamientos de los nodos del puente, estos desplazamientos son dinámicos, debido a las condiciones de las cargas permanentes, variables o accidentales, para definir estas cargas se realiza en el sitio de estudio la investigación de las fuerzas del viento, estadística de las cargas accidentales y además se realiza un aforo del número de peatones que transitan sobre la pasarela (Capra, 2005).

\subsubsection{Cargas por viento}

Las cargas por viento fueron medidas con un anemómetro digital, durante un periodo de doce meses, en horario de las 00:00 horas hasta las 24:00 horas, encontrando que los valores de las magnitudes de los vientos más representativos son en el mes de febrero, abril y mayo como se muestran en la Figura 3. Condición que servirá para definir los límites de medición y duración de la instrumentación (Li, 2014).

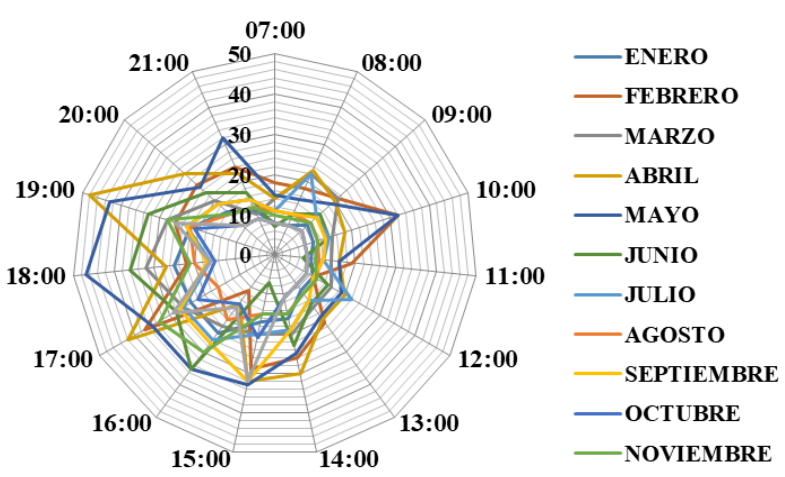

Figura 3: Representación gráfica de la magnitud del viento $[\mathrm{km} / \mathrm{hr}]$ en el sitio de estudio.

(Fuente Servicio Meteorológico Nacional UNAM)

\subsubsection{Cargas por sismo}

Las cargas por sismo fueron tomadas de la estadística del sistema sismológico nacional de la UNAM, durante un periodo de treinta y seis meses, en horario de las 00:00 horas hasta las 24:00 horas, encontrando que los valores de las magnitudes de los sismos más representativos son en el mes de septiembre, octubre y diciembre como se muestran en la Figura 4. 
Condición que sirven para definir los límites de medición y duración de la instrumentación.

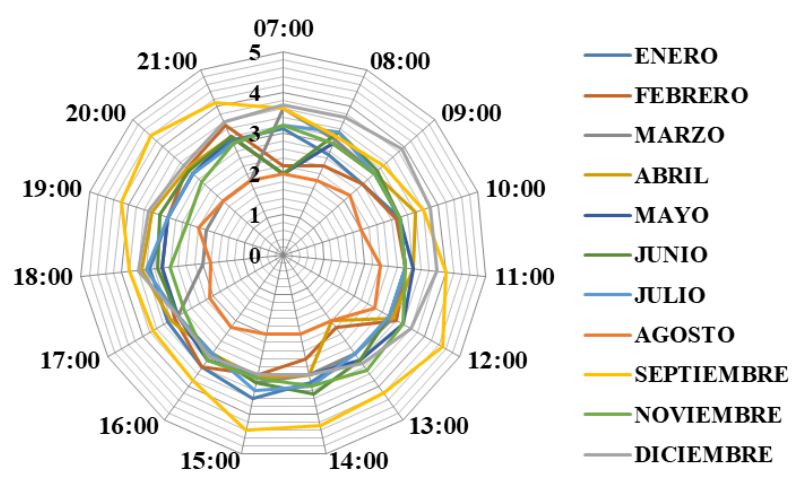

Figura 4: Representación gráfica de la magnitud de sismo [Escala Richter] en el sitio de estudio

(Fuente: Instrumental propio adquirido).

\subsubsection{Cargas vivas}

Las cargas vivas fueron determinadas realizando un aforo peatonal, durante un periodo de doce meses, en horario de las 00:00 horas hasta las 24:00 horas, encontrando que los valores de las magnitudes de los transeúntes más representativos son en el mes de julio, noviembre y diciembre como se muestran en la Figura 5. Condición que servirá para definir los límites de medición y duración de la instrumentación.

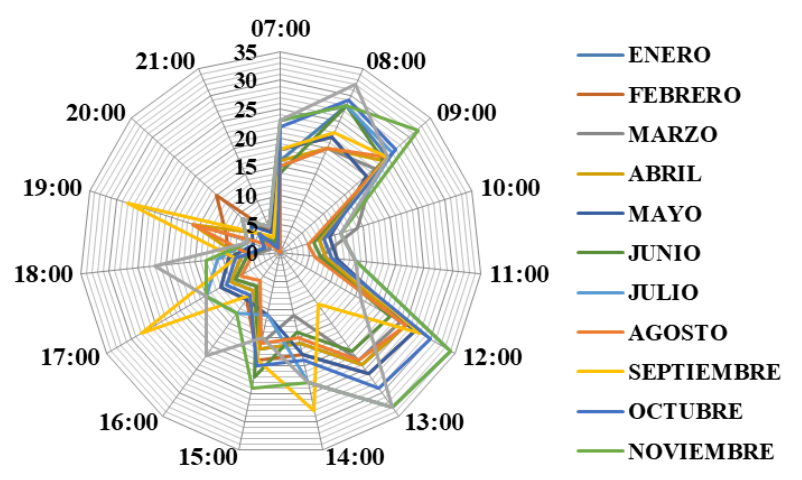

Figura 5: Representación gráfica del número de peatones que cruza el puente de ida y/o de regreso [personas] en el sitio de estudio. (Fuente: Documental propia medición en campo

\subsection{Selección de información.}

La información será seleccionada de acuerdo a tres factores importantes, el primero es definir la duración de la instrumentación, el segundo la definición de los límites temporales de la medición y el tercero es la capacidad de la base de datos útil para el análisis (Feldmann, 2010).

\subsubsection{Duración de la instrumentación.}

Los instrumentos son colocados en los puntos de control en la pasarela del puente durante tres periodos, el primero en el mes de febrero, abril y mayo en un horario de las 18:00 horas a las 19:00 horas, debido al análisis estadístico de las cargas por viento ver Figura 3, el segundo periodo en el mes de septiembre, octubre y diciembre en un horario de las 19:30 horas a las 20:30 horas, debido al análisis estadístico de las cargas por sismo ver Figura 4 y el tercer periodo en el mes de julio, noviembre y diciembre en un horario de las 12:00 horas a las 13:00 horas, debido al análisis estadístico de las cargas vivas ver Figura 5.

\subsubsection{Definición de los límites de medición.}

Los instrumentos miden la información de acuerdo a la duración de la instrumentación, la recepción de la información obtenida muestra valores de la variación de la velocidad en los ejes cartesianos, por tanto, se genera una base de datos enorme por que el instrumento almacena datos en forma temporal, es decir, existen datos por cada segundo medido (Ali, 2020).

Este proceso es cíclico, porque en el sitio de la investigación, las magnitudes de las cargas no son constantes durante todo el periodo de instrumentación debido a tres características de las fuerzas impuestas, la primera será cuando existe una ráfaga de viento, cuya duración promedio es de un minuto a dos minutos, tiempo observado durante la estadística de cargas de viento, la segunda será de acuerdo al periodo de las ondas sísmicas, cuyos datos estadísticos muestran periodos de 30 segundos, y la tercera será con el tiempo transcurrido de traslado del peatón por la pasarela del puente, en donde se observa una velocidad promedio de $1.5 \mathrm{~m} / \mathrm{s}$ (Ali, 2020), la longitud de la pasarela del puente mide $45.15 \mathrm{mts}$, por lo que una persona tarda 67.73 segundos en transitar de un extremo a otro, por consiguiente, se define la exploración experimental con duración de instrumentación continua de una hora y a partir de la información captada se selecciona con los límites temporales desde 0 segundos hasta 120 segundos con las magnitudes más representativas, este proceso es la corrida de datos diaria, este lapso de tiempo servirá para realizar la simulación numérica de cada evento experimental.

\subsubsection{Evento experimental.}

La información analizada posterior a definición de los límites de medición en cada duración de instrumentación, se compara diariamente, seleccionando las lecturas continuas más desfavorables, de tal manera que, al término se tendrá seleccionado solo una corrida de datos diaria, el evento experimental lo formaran cinco corridas de datos con duración de 120 segundos correspondientes a la instrumentación en cada punto de control (Alhassan, 2020).

\subsubsection{Base de datos.}

La información captada se almacena y se procesa en 5 carpetas correspondientes a los cinco puntos de control, dentro de cada carpeta se almacenan 9 archivos con información de cada periodo medido en los horarios establecidos durante los días programados. La información seleccionada se filtró de conformidad con la definición de los límites de medición y con la duración de la instrumentación. El filtro final de la información captada en los puntos de control corresponde a 
seleccionar solo un evento experimental y finalmente realizar la simulación (Salenko, 2021).

\subsection{Método de los desplazamientos}

Consiste en analizar los elementos estructurales mediante un matriz de rigidez, en donde se relaciona los desplazamientos de los nodos de la estructura, con las fuerzas exteriores provocadas por las cargas (Capra, 2005).

\subsubsection{Grados de libertad}

Los desplazamientos de los nodos o apoyos de una estructura, pueden ser desplazamientos lineales llamados traslaciones o desplazamientos angulares llamados rotaciones, el grado de libertad se representa con un número real y corresponde con la libertad que tiene un nodo para rotar o trasladarse, en este trabajo de investigación se analizaran las traslaciones en los ejes cartesianos a partir de la medición de la variación de la velocidad angular (Budipriyanto, 2015).

\subsection{Diseño estructural del puente}

Los grados de libertad corresponden a las posibles formas de moverse que tiene una estructura, con ellos se puede describir la figura deformada de una estructura como se observa en la Figura 6. Estos se miden en los puntos de unión de elementos (nudos) o en los apoyos al aplicarse el método de los desplazamientos

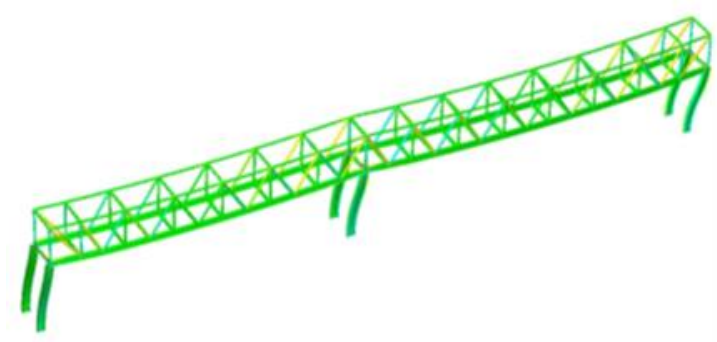

Figura 6: Representación gráfica de la figura deformada del puente
peatonal en el análisis estructural. peatonal en el análisis estructura

El diseño estructural logra que una estructura no falle en ningún momento de su vida útil. Se pueden presenciar diversos tipos de falla, como es el caso del límite de servicio, que ocurre en el momento que la estructura presenta deformaciones excesivas, es decir, que los nodos tengan tal desplazamiento que se detecte inestabilidad para los peatones al momento de cruzar la pasarela en este caso lo llamaremos riesgo de servicio (Capra, 2005) (Zheng, 2018).

La falla por resistencia se detecta cuando existe una separación de las partes de la estructura, debido a fatiga que producen las cargas cíclicas, es decir, la estructura del puente está sometida a repeticiones de carga, el diseño estructural predice la magnitud de los desplazamientos generados por estas cargas y dispone de las condiciones para resistirlas, determinando la variación de la velocidad en cada nodo de la estructura de forma conjunta, este proceso es llamado modo de vibrar (Alhassan, 2020), tal como se presenta en la Tabla 2.

Los elementos del puente deben soportar todas las cargas y sus combinaciones, las cargas producen deformaciones de dos tipos, las primeras deformaciones elásticas, es decir, cuando los esfuerzos y las deformaciones forman una línea recta, en este caso se presenta el riesgo elástico y las segundas son cuando las cargas producen deformaciones inelásticas, es decir cuando las deformaciones no desaparecen en cualquier valor de los esfuerzos, además, las uniones o la geometría de los elementos estructurales del puente tienen una falla interna no detectable a simple vista, en este caso se presenta el riesgo inelástico (Capra, 2005).

Tabla 2: Modos de falla de la estructura del puente (Fuente: Elaboración propia)

\begin{tabular}{c|c}
$\begin{array}{c}\text { Modo de } \\
\text { Vibrar }\end{array}$ & Aceleración \\
\hline 1 & {$[\mathbf{m} / \mathbf{s} 2]$} \\
2 & 0.38 \\
3 & 0.38 \\
4 & 0.38 \\
5 & 0.31 \\
6 & 0.27 \\
& 0.17 \\
\hline
\end{tabular}

\subsection{Variación de la velocidad}

El movimiento de los nodos se observa con la magnitud de los desplazamientos del análisis estructural al aplicar el método de los desplazamientos, los nodos a lo largo de la secuencia del paso del tiempo, pueden tener una trayectoria $\mathrm{u}$ órbita, con formas rectilínea, curva o parabólica, esta trayectoria forma un sistema de desplazamientos nodales en el espacio a lo largo del tiempo, por consecuencia, los nodos cambian de posición a una cierta velocidad. La variación de la velocidad con respecto al tiempo se denomina aceleración, y su trayectoria curva define la velocidad angular, la aceleración permitida para disminuir el riesgo de servicio, el riesgo elástico y el riesgo inelástico, debe cumplir estándares de aceptación al aplicar los reglamentos (Feldmann, 2010) (Capra, 2005) (Shi, 2018).

\section{Resultados y discusión}

Se observó que los dos tramos A-C y C-E, tienen diferente comportamiento, en la medición del eje cartesiano " $\mathrm{x}$ ", en el primer tramo la máxima magnitud de la aceleración, está ubicada a los 35 segundos como se observa en la figura 7, lo que representa una respuesta dinámica de la estructura, por consiguiente, en el apoyo "B", se tiene la máxima respuesta de vibraciones. 


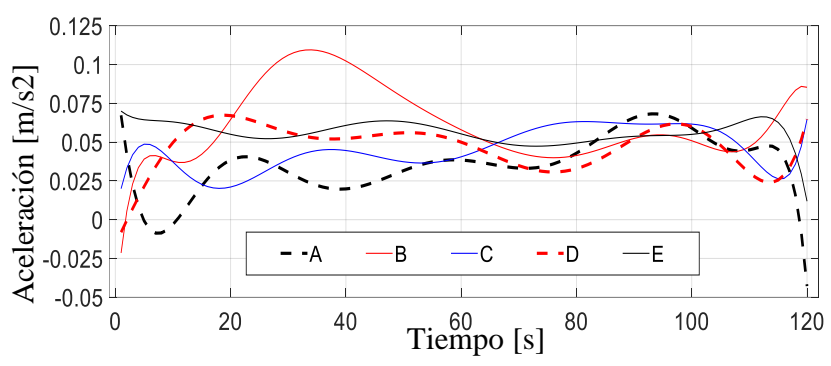

Figura 7: Representación gráfica de la aceleración en el eje " $\mathrm{X}$ " (Fuente: Elaboración propia)

En el punto "B" no se espera posible daño porque la magnitud de las aceleraciones es menor que la norma.

En relación a las vibraciones en el eje " $z$ ", transversal a la superficie de transitado, se observa que en la figura 8 , las condiciones en los elementos estructurales, son similares con respecto del eje "x", existe un incremento de la magnitud de la aceleración en el punto " $D$ ", a tal grado que representa desplazamientos similares que el punto "B", ambos mayores que en el resto de los puntos de control.

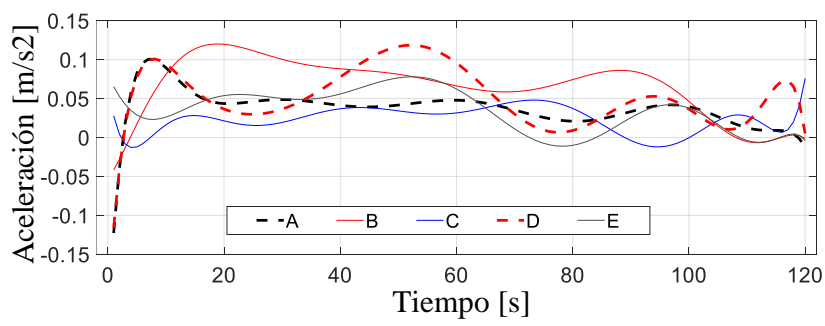

Figura 8: Representación gráfica de la aceleración en el eje " $Z$ " (Fuente: Elaboración propia)

Los mayores desplazamientos medidos se observan en el eje " $y$ ", ver figura 9, se observa en el nodo de control " $A$ " que su respuesta alcanza magnitudes de $0.68 \mathrm{~m} / \mathrm{s}^{2}$, en el nodo "B" alcanza $0.35 \mathrm{~m} / \mathrm{s}^{2}$, siendo ambos comportamientos mayores del comportamiento del resto de los puntos de control.

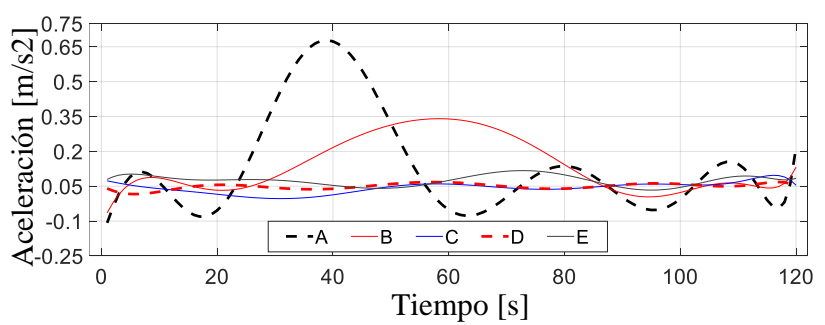

Figura 9: Representación gráfica de la aceleración en el eje "Y" (Fuente: Elaboración propia)

El riesgo inelástico es un factor limitante de fallos, es decir, las aceleraciones de la respuesta de la estructura del puente no deben ser mayores al riesgo inelástico, en caso contrario, el puente tendría inestabilidad estructural (Capra, 2005).

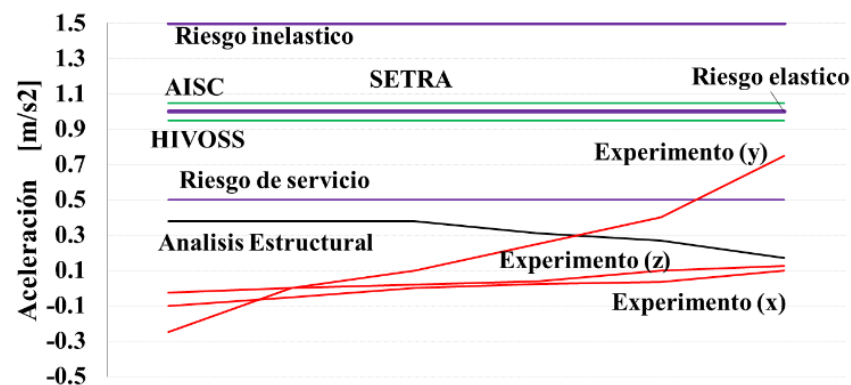

Figura 10: Representación gráfica de la comparación de la aceleración. (Fuente: Norma HIVOSS, AISC, SETRA,

Documental propio de campo)

En la Figura 10 se presenta la magnitud de la aceleración del riesgo de servicio, el riesgo elástico y el riesgo inelástico, los valores de la normatividad HIVOSS, SETRA y AISC (Gheitasi, 2016) (Salenko, 2021), de igual forma, se observan los valores del evento experimental en los ejes cartesianos (x, y, z) y la magnitud de la aceleración de los modos de vibrar del análisis estructural dinámico del puente peatonal (Zheng, 2018).

\section{Conclusión}

La instrumentación y medición de aceleraciones en los puntos de control muestran el comportamiento en tiempo real del sistema estructural y posibilita la toma de decisiones para cambiar la respuesta dinámica en forma local o general, en este trabajo de investigación se realizó la medición de las aceleraciones en la superficie de la pasarela, aplicando técnicas de instrumentación, medición y procesamiento. La obtención de resultados implementando la metodología concluye con las ventajas siguientes:

1. El modelo considera la historia de la aceleración en la pasarela, generando resultados cuya magnitud es inferior a los normados, reportado por los autores.

2. La simulación muestra resultados muy aproximados a los presentados en la literatura, además, estos, son significativos al momento de evaluarlos, debido a que la magnitud del riesgo se compara con las normas vigentes.

3. Basándose en la metodología y los resultados obtenidos, es menester realizar como trabajo futuro un seguimiento detallado de inspección en el nodo "A", con respecto a que presenta aceleraciones cuya magnitud se encuentra en la zona de riesgo de servicio, anticipándose por medio de procedimientos constructivos para cambiar la respuesta dinámica y disminuir las aceleraciones.

4. La simulación muestra magnitudes del análisis estructural por debajo del riesgo de servicio, sin embargo, no es así en comparación con las magnitudes del evento experimental en los puntos de control.

5. La presente investigación concluye que los elementos estructurales del puente se encuentran con estabilidad estructural, debido a que la respuesta dinámica se encuentra en la zona de riesgo elástico. 


\section{Agradecimientos}

Los autores desean expresar su agradecimiento a la Universidad Autónoma del Estado de Hidalgo por su apoyo para la realización de la presente investigación.

\section{Referencias}

Alhassan, M. A. (2020). Control of Vibrations of Common Pedestrian Bridges in Jordan Using Tuned Mass Dampers. Procedia Manufacturing, 44(2019), 36-43. https://doi.org/10.1016/j.promfg.2020.02.202

Ali, S. (2020). Numerical study of pedestrian suspension bridge with innovative composite deck. Heliyon, 6(7), e04473.

https://doi.org/10.1016/j.heliyon.2020.e04473

Banerjee, A. (2020). A comparative study of pedestrian movement behavior over foot over bridges under similar land-use type. Transportation Research Procedia, 48(2019), 3342-3354. https://doi.org/10.1016/j.trpro.2020.08.119

Budipriyanto, A. (2015). Dynamic responses of a steel railway bridge for the structure's condition assessment. Procedia Engineering, 125, 905-910. https://doi.org/10.1016/j.proeng.2015.11.085

Capra. (2005). Vulnerabilidad de edificaciones e infraestructura. Informe Técnico ERN-CAPRA, 48.

Feldmann, M. (2010). Human-induced vibration of steel structures (Hivoss) background document -, 24183. Retrieved from

http://dx.doi.org/10.2777/79056

Gheitasi, A. (2016). Experimental and analytical vibration serviceability assessment of an in-service footbridge. Case Studies in Nondestructive Testing and Evaluation, 6, 79-88.

https://doi.org/10.1016/j.csndt.2016.11.001

Li, Y. F. (2014). Case study of first all-GFRP pedestrian bridge in Taiwan. Case Studies in Construction Materials, 1, 83-95. https://doi.org/10.1016/j.cscm.2014.05.001

Salenko. (2021). Strength, Flexural Rigidity and Aerodynamic Stability of Fiberglass Spans in Pedestrian Suspension Bridge. Transportation Research Procedia, 54(2020), 758-767.

https://doi.org/10.1016/j.trpro.2021.02.131

SETRA. (2006). Footbridges - Assessment of vibrational behaviour of footbridges under pedestrian loading, (october), 127.

Shi, W. (2018). Application of an artificial fish swarm algorithm in an optimum tuned mass damper design for a pedestrian bridge. Applied Sciences (Switzerland), 8(2). https://doi.org/10.3390/app8020175

Zheng, J. (2018). Concrete-Filled Steel Tube Arch Bridges in China. Engineering, 4(1), 143-155. https://doi.org/10.1016/j.eng.2017.12.003 